\title{
Prácticas profesionales en el área de salud: Retos y Consecuencias afrontadas en el marco de la pandemia por Covid-19
}

\author{
Professional practices in the health area: \\ Challenges and Consequences faced in the \\ framework of the Covid-19 pandemic
}

\section{Práticas profissionais na área da saúde: Desafios e consequências enfrentados no quadro da pandemia Covid-19}

Deisy Pinto*

Fernando Santana De Paiva*

Resumen: El presente documento corresponde a un estado del arte en el que se examinan los retos que afronta el profesional que labora en el área de salud, en el marco de la pandemia generada por el Covid-19. Se realizó búsqueda bibliográfica en las siguientes bases de datos: Scielo, Redalyc, Dialnet y Google scholar, encontrándose una muestra final de 40 artículos. A partir de la lectura y abordaje analítico de las publicaciones realizadas sobre la temática se deducen 3 categorías de análisis: 1) Política Pública de Salude y Covid-19; 2) Condiciones de trabajo y efectos en la salud de los profesionales y 3 ) efecto en la salud mental de los profesionales. Los resultados de la presente revisión sistemática arrojan que los retos afrontados afectan con serias consecuencias al personal de salud, tanto a nivel físico, como mental y social.

Palabras claves: Profesionales y políticas públicas. Políticas públicas y pandemia. Profesionales de la salud y Covid-19. Salud mental y covid-19.

Abstract: This document corresponds to a state of the art in which the challenges faced by professionals working in the health area are examined, in the framework of the pandemic generated by the Covid-19. A bibliographic

\footnotetext{
*Estudiante de Maestria, Universidad Federal Juiz de Fora (Brasil). Especialista en Psicología Clínica egresada de la Universidad San Buenaventura (Cali-Colombia). Psicóloga, egresada de la Universidad del Valle (Cali-Colombia). E-mail pinto612@gmail.com.

** Doctorado en psicología. Docente de la Univerversidad Federal Juiz de Fora (UFJF) Orientador de Investigaciones a estudiantes de Maestría y Doctorado UFJF. E-mail: fernandosantana.paiva@gmail.com.
} 
search was carried out in the following databases: Scielo, Redalyc, Dialnet and Google Scholar, finding a final sample of 40 articles. From the reading and analysis of the publications made on the subject, 3 categories of analysis are deduced: 1) Public Health Policy and Covid-19; 2) Working conditions and effects on the health of professionals; and 3) effect on the mental health of professionals. The results of this systematic review show that the challenges faced affect health professionals with serious consequences, both physical, mental and social.

Key words: Professionals and public policies. Public policies and pandemic. Health professionals and Covid-19. Mental health and covid-19.

Resumo: Este documento corresponde a um estado da arte no qual são examinados os desafios enfrentados pelo profissional que atua na área da saúde, no quadro da pandemia gerada pela Covid-19. Realizou-se um levantamento bibliográfico nas seguintes bases de dados: Scielo, Redalyc, Dialnet e Google scholar, encontrando uma amostra final de 40 artigos. Da leitura e abordagem analítica das publicações realizadas sobre o tema, deduzem-se 3 categorias de análise: 1) Políticas Públicas de Saúde e Covid-19; 2) Condições de trabalho e efeitos na saúde dos profissionais e 3) efeito na saúde mental dos profissionais. Os resultados da presente revisão sistemática mostram que os desafios enfrentados afetam os profissionais de saúde com graves consequências, tanto física, mental e social.

Palavras-chave: Profissional e políticas publicas. Políticas publicas e pandemia. Profissionais da saúde e Covid-19. Saúde mental e covid-19.

Recebido em 05/10/2021. Aceito em 20/06/2021:

\section{Introducción}

El llamado virus Covid-19, cepa del coronavirus SARS-CoV-2, que afecta las vías respiratorias mediante problemas de neumonía, inició en China a finales del año 2019 y se propagó por todo el mundo hasta convertirse en una Pandemia, cobrando hasta el momento actual la vida de millones de personas (SAMANIEGO et al; 2020). La Organización Mundial de la Salud (OMS) declaró el 30 de enero del 2020 emergencia en Salud a nivel internacional y el 11 de marzo del mismo año diagnosticó el brote como pandemia (MÁRQUEZ, 2020; PEREIRA et al., 2020; RAMOS; AMBROSIO, 2020; URZÚA, 2020).

En el año 2020, del mes de abril, se reportaron 2.500 casos de contagio en todo el mundo (GONZALES et al., 2020), al mes de agosto, eran más de 700 las personas fallecidas a nivel mundial y 19.000.000 las infectadas, 10.5 millones pertenecientes a las américas (URZUA et al., 2020); a enero del 2021 son 1,861,005 defunciones reportadas (LANDEROS; ARROYO y RODRÍGUEZ, 2020). De manera global, salud pública para reducir el impacto causado por el virus y evitar el contagio, ha optado por la implementación de medidas de protección y prevención, tales como, lavado de 
manos, distanciamiento social, cuarentena, uso de cubrebocas (BRIONES et al., 2020; GONZALES et al., 2020; LAZCANO y ALPUCHE, 2020; PEREIRA et al., 2020; SAMANIEGO et al; 2020; URZÚA et al., 2020). No obstante, pese a todos los esfuerzos realizados y medidas de protección, no ha sido posible la detención de la pandemia, además de tenerse incertidumbre al respecto.

De acuerdo con lo anterior, a nivel mundial se ha sentido el fuerte impacto que genera el nuevo virus, reportándose por los diversos medios las consecuencias físicas, sociales y económicas padecidas por la población, siendo urgente, también, particularizar el asunto sobre los profesionales de la salud, ellos obligatoriamente están sometidos a extenuantes retos en el ejercicio de su rol profesional. A partir de pesquisas realizadas en los distintos continentes y del escenario de pandemia que azota actualmente el mundo, se pueden inferir los retos que afronta el personal del área de salud (médicos, enfermeros, fisioterapeutas, odontólogos, psicólogos etc.). Los desafíos más recurrentes son: laborar en ausencia de abastecimiento de equipos protectores de bioseguridad, brindar atención a personal infectado por Covid-19, mayor riesgo de muerte ante el contagio, adaptación al fatigante contexto laboral, estigmatización social, aprender a autocontrolarse emocionalmente para cumplir con la atención a usuarios.

Se evidencian también que el profesional de la salud en mayor medida manifiesta síntomas asociados con estrés, insomnio, depresión, ansiedad y solo tres autores de la literatura revisada reportaron casos de temor a ser agredidos a consecuencia de la estigmatización social. Lo anterior cobra relevancia a partir de investigaciones realizadas a lo largo de diversos continentes, en países como Paraguay, Chile, Brasil, Colombia, China, Rumania, Alemania y Ecuador, Italia, Inglaterra. Esta situación, afecta drásticamente la salud no solo por las consecuencias físicas que experimentan los profesionales ante el contagio, también a nivel mental y social, es decir, un impacto biopsicosocial a raíz del trauma psicosocial que atraviesan.

Por lo expuesto anteriormente surge el interés de realizar una revisión sistemática mediante el estado del arte sobre las Prácticas profesionales en el área de salud: Retos y Consecuencias afrontadas en el marco de la pandemia por Covid-19

\section{Metodología}

La presente revisión bibliográfica facilita en primer lugar, la apreciación y visibilización de lo acontecido a nivel internacional sobre la temática tratada, segundo, identifica propuestas relevantes a nivel internacional, desde el punto de vista de salud pública y de investigadores, para minimizar o dar tratamiento al impacto generado. La revisión de la literatura fue realizada en las bases de datos Scielo, Redalyc, Dialnet y Google scholar, para la búsqueda se recurrió a palabras claves tales como: Profesionales y políticas públicas, Políticas públicas y pandemia, Profesionales de la Salud y Covid-19, salud mental y pandemia, aplicándose como criterios de inclusión: A) Literatura en el idioma español y portugués; B) Investigaciones sobre el tema propuesto provenientes de diversos países; C) Revisiones teóricas y hallazgos de investigaciones sobre las diferentes prácticas profesionales en el contexto de la pandemia y el rol de salud pública; D) pesquisas sobre Salud Mental en profesionales de la salud durante la pandemia; E) Tesis y monografías. Se excluyeron las publicaciones cuyo objeto de investigación no eran los profesionales de la salud ni el impacto a nivel de salud mental por causa del Covid-19, o publicaciones no pertenecientes a esta área.

Se obtuvieron 1.638 artículos y luego se prosiguió con la revisión del material preseleccionando 143 documentos que parecían ser afín con nuestro tema. Luego de la revisión en el paso anterior, se descartan 103 publicaciones por no contar con los criterios de inclusión anteriormente 
mencionados, especialmente porque su foco no eran las prácticas profesionales en salud en el escenario de la pandemia, seleccionándose así 40 artículos, para su posterior lectura completa y análisis final (Ver figura 1).

Figura 1. Procedimiento para selección bibliográfica

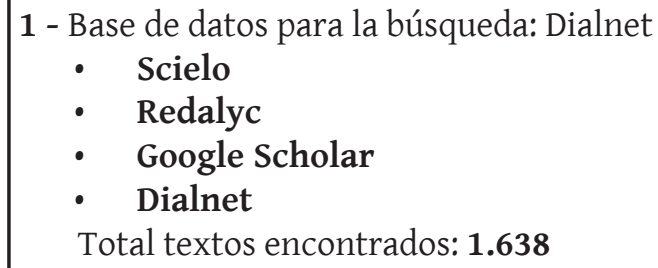

2 - Criterios de inclusión para la búsqueda:

A) Literatura en el idioma español y portugués;

B) Investigaciones sobre el tema provenientes de diversos países;

C) Revisiones teóricas y hallazgos de investigaciones sobre las diferentes prácticas profesionales en el contexto de la pandemia y el rol de salud pública; D) Pesquisas sobre Salud Mental en profesionales de la salud durante la pandemia.

E) Tesis y monografías.

5 - Textos descartados
luego de la revisión del
paso 3: 103
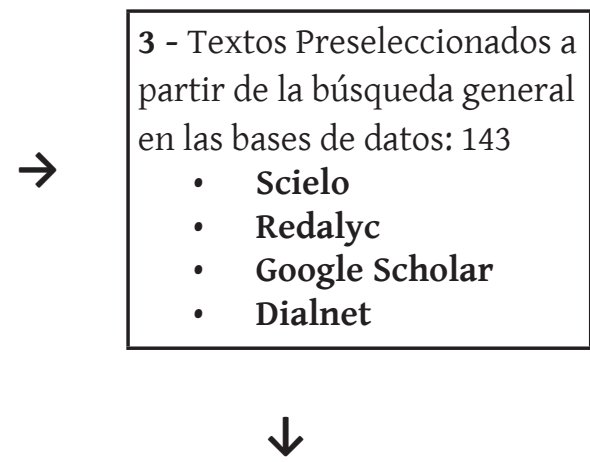

4 - Total textos seleccionados para análisis a profundidad: 40

Fuente: Los autores.

\section{Resultados}

\section{Datos Bibliométricos}

Los 40 artículos seleccionados para análisis se sintetizan en el cuadro 1, agrupándose los títulos encontrados por cada categoría de análisis. Cabe aclarar que 2 de los artículos (En cursiva) son citados fuera de las mencionadas categorías. Los planteamientos de los diversos autores pueden estar distribuidos a lo largo de las categorías sin haber restricción en cada una de ellas por autor. Se resalta que en el proceso de búsqueda y en función a los parámetros estipulados se encontraron publicaciones sobre el tema que en su mayoría correspondían al año 2020 (38), solo se encontraron 2 publicaciones correspondientes al año en curso. (Ver cuadro 1). 
Cuadro 1: Síntesis Bibliométrica.

\begin{tabular}{|c|c|c|c|}
\hline \multirow[t]{2}{*}{ Título encontrado por categoría } & \multicolumn{3}{|c|}{ Categorías de análisis } \\
\hline & $\begin{array}{l}\text { Política } \\
\text { Pública } \\
\text { de Salud y } \\
\text { Covid-19 }\end{array}$ & $\begin{array}{l}\text { Condiciones de } \\
\text { trabajo y efectos } \\
\text { en la salud de los } \\
\text { profesionales en } \\
\text { el afrontamiento } \\
\text { al Covid-19 }\end{array}$ & $\begin{array}{l}\text { Impacto en la } \\
\text { salud mental } \\
\text { del personal } \\
\text { de salud como } \\
\text { consecuencia } \\
\text { de los retos } \\
\text { afrontados. } \\
\end{array}$ \\
\hline $\begin{array}{l}\text { Protección de los profesionales sanitarios en } \\
\text { nefrología ante la pandemia por COVID- } 19 \text {. }\end{array}$ & & & $\mathrm{X}$ \\
\hline $\begin{array}{l}\text { CORONAVIRUS (COVID-19) Y SU IMPACTO EN } \\
\text { LA SALUD MENTAL DE LOS PROFESIONALES DE } \\
\text { LA SALUD EN ANTIOQUIA }\end{array}$ & & & $\mathrm{X}$ \\
\hline $\begin{array}{l}\text { La COVID-19 y los desafíos que impone para el } \\
\text { profesional de la salud en Cuba }\end{array}$ & $\mathrm{X}$ & & \\
\hline $\begin{array}{l}\text { APLICACIÓN DE NORMAS DE BIOSEGURIDAD } \\
\text { EN EL PERSONAL DE ENFERMERÍA EN TIEMPOS } \\
\text { DE PANDEMIA EN EL HOSPITAL BASICO } \\
\text { PELILEO }\end{array}$ & & $\mathrm{X}$ & \\
\hline $\begin{array}{l}\text { La pandemia del maltrato contra el personal de } \\
\text { la salud en tiempos de pandemia. }\end{array}$ & & & $\mathrm{X}$ \\
\hline $\begin{array}{l}\text { Intervenções em saúde mental para } \\
\text { profissionais de saúde frente a pandemia de } \\
\text { Coronavirus. }\end{array}$ & & & $\mathrm{X}$ \\
\hline $\begin{array}{l}\text { Luz ultravioleta para desinfección en áreas de } \\
\text { salud, frente al Covid-19. Revisión de literatura. }\end{array}$ & & $\mathrm{X}$ & $\mathrm{X}$ \\
\hline $\begin{array}{l}\text { Cuidar al que cuida: el impacto emocional de la } \\
\text { epidemia de coronavirus en las enfermeras y } \\
\text { otros profesionales de la salud }\end{array}$ & & & $\mathrm{X}$ \\
\hline $\begin{array}{l}\text { Ataque al personal de la salud durante la } \\
\text { pandemia de COVID-19 en Latinoamérica }\end{array}$ & & $\mathrm{X}$ & $\mathrm{X}$ \\
\hline $\begin{array}{l}\text { Enfermería y COVID-19: reconocimiento de la } \\
\text { profesión en tiempos de adversidad }\end{array}$ & & $\mathrm{X}$ & $\mathrm{X}$ \\
\hline $\begin{array}{l}\text { Pandemia de la COVID-19 y las Políticas de } \\
\text { Salud Pública en el Perú }\end{array}$ & $\mathrm{X}$ & & $\mathrm{X}$ \\
\hline $\begin{array}{l}\text { Psicologia Hospitalar e da Saúde no } \\
\text { enfrentamento do coronavírus: necessidade e } \\
\text { proposta de atuação }\end{array}$ & & & $\mathrm{X}$ \\
\hline $\begin{array}{l}\text { CoVID-19. Un reto para los profesionales de la } \\
\text { salud }\end{array}$ & $\mathrm{X}$ & & \\
\hline $\begin{array}{l}\text { La necesidad de nuevos paradigmas de } \\
\text { cooperación y riesgo de los trabajadores de la } \\
\text { salud. }\end{array}$ & $\mathrm{X}$ & & \\
\hline ¿Será mejor la salud pública tras la COVID-19? & $\mathrm{X}$ & & \\
\hline
\end{tabular}


continuação

\begin{tabular}{|c|c|c|c|}
\hline \multirow[t]{2}{*}{ Título encontrado por categoría } & \multicolumn{3}{|c|}{ Categorías de análisis } \\
\hline & $\begin{array}{l}\text { Política } \\
\text { Pública } \\
\text { de Salud y } \\
\text { Covid-19 }\end{array}$ & $\begin{array}{l}\text { Condiciones de } \\
\text { trabajo y efectos } \\
\text { en la salud de los } \\
\text { profesionales en } \\
\text { el afrontamiento } \\
\text { al Covid-19 }\end{array}$ & $\begin{array}{l}\text { Impacto en la } \\
\text { salud mental } \\
\text { del personal } \\
\text { de salud como } \\
\text { consecuencia } \\
\text { de los retos } \\
\text { afrontados. } \\
\end{array}$ \\
\hline $\begin{array}{l}\text { Síndrome de Burnout en personal de salud } \\
\text { durante la pandemia COVID-19 }\end{array}$ & & & $\mathrm{X}$ \\
\hline $\begin{array}{l}\text { Fortalezas, deficiencias y respuestas del } \\
\text { sistema nacional de salud frente a la Pandemia } \\
\text { del Covid-19 }\end{array}$ & $\mathrm{X}$ & & $\mathrm{X}$ \\
\hline $\begin{array}{l}\text { Formulación de políticas públicas en salud durante } \\
\text { la pandemia de covid-19 en países americanos }\end{array}$ & & & \\
\hline $\begin{array}{l}\text { Alfabetización en salud pública ante la emergencia } \\
\text { de la pandemia por Covid-19 Salud pública de } \\
\text { México }\end{array}$ & & & \\
\hline $\begin{array}{l}\text { ¿Qué papel desempeña la Fisioterapia en la } \\
\text { pandemia Mundial por COVID-19? }\end{array}$ & & & $\mathrm{X}$ \\
\hline $\begin{array}{l}\text { Impacto de la epidemia del Coronavirus } \\
\text { (COVID-19) en la salud mental del personal de } \\
\text { salud y en la población general de China }\end{array}$ & & & $\mathrm{X}$ \\
\hline $\begin{array}{l}\text { El síndrome de Burnout en los profesionales de } \\
\text { salud en la pandemia por la COVID-19 }\end{array}$ & & & $\mathrm{X}$ \\
\hline $\begin{array}{l}\text { Cuidado y autocuidado en el personal de salud: } \\
\text { enfrentando la pandemia COVID-19 }\end{array}$ & & & $\mathrm{X}$ \\
\hline $\begin{array}{l}\text { Teleconsulta en la pandemia por Coronavirus: } \\
\text { desafíos para la telemedicina pos-COVID-19 }\end{array}$ & $\mathrm{X}$ & & $\mathrm{X}$ \\
\hline $\begin{array}{l}\text { Necesidad de apoyo psicológico en } \\
\text { profesionales sanitarios ante el COVID-19 }\end{array}$ & & & $\mathrm{X}$ \\
\hline $\begin{array}{l}\text { A luta dos professionais de saúde no } \\
\text { enfrentamento da COVID-19 }\end{array}$ & & & $\mathrm{X}$ \\
\hline $\begin{array}{l}\text { Conocimientos y necesidades del personal de } \\
\text { salud sobre elementos de protección personal } \\
\text { en el departamento del Cauca, durante la } \\
\text { pandemia por coronavirus COVID-19. }\end{array}$ & & & $\mathrm{X}$ \\
\hline $\begin{array}{l}\text { Estrés laboral, ansiedad y miedo al COVID-19 } \\
\text { en médicos generales colombianos }\end{array}$ & & & $\mathrm{X}$ \\
\hline $\begin{array}{l}\text { Síndrome de Burnout y relación con los } \\
\text { factores sociodemográficos y laborales en } \\
\text { profesionales de la salud en una clínica privada } \\
\text { de la ciudad de Cuenca en el contexto con la } \\
\text { emergencia sanitaria por Covid-19 }\end{array}$ & & & $\mathrm{X}$ \\
\hline
\end{tabular}


conclusão

\begin{tabular}{|c|c|c|c|}
\hline \multirow[t]{2}{*}{ Título encontrado por categoría } & \multicolumn{3}{|c|}{ Categorías de análisis } \\
\hline & $\begin{array}{l}\text { Política } \\
\text { Pública } \\
\text { de Salud y } \\
\text { Covid-19 }\end{array}$ & $\begin{array}{l}\text { Condiciones de } \\
\text { trabajo y efectos } \\
\text { en la salud de los } \\
\text { profesionales en } \\
\text { el afrontamiento } \\
\text { al Covid-19 }\end{array}$ & $\begin{array}{l}\text { Impacto en la } \\
\text { salud mental } \\
\text { del personal } \\
\text { de salud como } \\
\text { consecuencia } \\
\text { de los retos } \\
\text { afrontados. } \\
\end{array}$ \\
\hline $\begin{array}{l}\text { Salud laboral frente a la pandemia del } \\
\text { COVID-19 }\end{array}$ & & & $\mathrm{X}$ \\
\hline $\begin{array}{l}\text { FISIOTERAPIA Y SU RETO FRENTE AL } \\
\text { COVID- } 19 .\end{array}$ & & & $\mathrm{X}$ \\
\hline $\begin{array}{l}\text { Actualidades de la educación médica cubana } \\
\text { frente a la COVID-19 }\end{array}$ & & & $\mathrm{X}$ \\
\hline $\begin{array}{l}\text { Reações e sentimentos dos profissionais de } \\
\text { saúde no cuidado de pacientes hospitalizados } \\
\text { com suspeita de Covid-19 }\end{array}$ & $\mathrm{X}$ & & $\mathrm{X}$ \\
\hline $\begin{array}{l}\text { Próximos pasos para fortalecer los Sistemas } \\
\text { de Salud y Atención Social del Reino Unido } \\
\text { a partir de las debilidades reveladas por la } \\
\text { pandemia de coronavirus }\end{array}$ & $\mathrm{X}$ & & \\
\hline $\begin{array}{l}\text { Sintomatología asociada a Trastornos de Salud } \\
\text { Mental en trabajadores sanitarios en Paraguay: } \\
\text { Efecto COVID-19 }\end{array}$ & & & $\mathrm{X}$ \\
\hline $\begin{array}{l}\text { Os trabalhadores de enfermagem na pandemia } \\
\text { Covid-19 e as desigualdades sociais. }\end{array}$ & & & $\mathrm{X}$ \\
\hline $\begin{array}{l}\text { TÍTULO: SINDROME DE BURNOUT EN EL } \\
\text { PERSONAL DE SALUD QUE TRABAJA EN LA } \\
\text { UNIDAD DE TERAPIA INTENSIVA DURANTE } \\
\text { LA PANDEMIA COVID-19 EN UN HOSPITAL DE } \\
\text { QUITO }\end{array}$ & & & $\mathrm{X}$ \\
\hline $\begin{array}{l}\text { Salud mental en trabajadores de la salud } \\
\text { durante la pandemia por COVID-19 }\end{array}$ & $\mathrm{X}$ & & $\mathrm{X}$ \\
\hline $\begin{array}{l}\text { LA BIOSEGURIDAD Y EL PERSONAL DE SALUD: } \\
\text { A PROPÓSITO DE LA PANDEMIA DE COVID-19 }\end{array}$ & $\mathrm{X}$ & & $\mathrm{X}$ \\
\hline $\begin{array}{l}\text { Enfermería en Colombia en tiempos de la } \\
\text { pandemia por coronavirus }\end{array}$ & & & $\mathrm{X}$ \\
\hline
\end{tabular}

Fuente: Los autores

Se puede visualizar también que los artículos de publicación recopilados para análisis, correspondientes en su 98\% a trabajos realizados durante el año 2020, se concentran en 3 categorías que reflejan los temas, sobre el asunto, que han sido relevantes para los académicos y sobre las cuales han publicado: la C1 (Política Pública de Salude y Covid-19) que está conformada por 11 autores que a ella se refieren; la C2 (Condiciones de trabajo y efectos en la salud de los profesionales en el afrontamiento al Covid-19) construida por los planteamientos de 4 autores y la C3 (Efecto en 
la salud mental del personal de salud como consecuencia de los retos afrontados) está compuesta por 32 autores de referencia. De tal forma que, publicaciones realizadas se concentran en el personal de salud y en las consecuencias de la pandemia por Covid-19 a nivel de salud mental.

La población objeto de estudio abordada fueron predominantemente médicos y enfermeros, seguido de psicólogos, odontólogos, fisioterapeutas, quienes también han sido llamados como profesionales de primera línea. Los diversos países en que se desarrollaron las investigaciones pertenecen a América latina, Asia y Europa, en países como Paraguay, Chile, Brasil, Colombia, China, Rumania, Alemania y Ecuador y en menor medida Italia, Islandia y Nueva Zelanda.

\section{Política Pública de Saluds y Covid-19.}

De forma particular, cada país del mundo decretó su estado de emergencia ante el incremento de infectados y muertes. Así, por ejemplo, Perú desde el 15 de marzo inicia sus medidas de seguridad cerrando actividades económicas, control de salidas a las calles y aislamiento social, todo con serias consecuencias económicas y sociales (GONZALES et al., 2020). Por su parte, el gobierno de Suecia, sugirió que el virus se debe propagar para poder alcanzar la inmunidad colectiva, pues los expertos han indicado que la pandemia se detiene cuando el 60-90\% de la población la padezca.

Otros países, como China y Corea del Sur, hicieron uso de la estrategia de supresión, que es efectiva cuando se combina, primero, con su implementación temprana, segundo, con una sólida estructura económica y tercero, con la contribución de la población (GONZALES et al., 2020). Los países mencionados aplicaron esta estrategia, a la vez que hicieron tempranamente seguimiento riguroso, aislaron rápidamente la población infectada, decretaron inmovilidad social obligatoria, detención de casos asintomáticos y aislamiento de casos nuevos (GONZALES et al., 2020).

Italia y España siguieron el modelo de China, no obstante, la capacidad del sistema de salud colapsó, al igual que en Perú (GONZALES et al., 2020) y muchos países más. En el caso de Colombia se decretaron varias medidas de protección a la población, entre ellas: el uso obligatorio de tapabocas, aislamiento obligatorio definido, se reglamentó el uso de la Telemedicina (Asesoría clínica virtual), para reducir el riesgo de contagios e incrementar el acceso a los servicios de salud, estrategia virtual también usada en Canadá, como soporte para la atención médica y refuerzo al sistema de salud (MÁRQUEZ, 2020).

En México se implementó como estrategia el distanciamiento social y la cuarentena, además de apostar a la innovación tecnológica, con la fabricación de sus propios equipos de protección personal (EPP) para el área de salud y usuarios (KERSHENOBICH, 2020). En Paraguay, como estrategia optaron por control de viajeros, suspensión de reuniones públicas, actividades académicas, previendo que el personal de salud no daría abasto para la atención, por el previsible colapso del sistema sanitario (URZÚA, 2020). En Cuba, gestionaron estrategias tempranas de detección, respuestas médicas rápidas, aislamientos, inmovilización social, cierre de instituciones, cuarentenas, reordenamiento laboral, investigaciones e incorporación de estudiantes a pesquisas en las que buscan en cada residencia a pacientes infectados, dictan charlas sobre prácticas de autocuidado y visitan ancianos que viven solos, adicionalmente, 2041 personal de salud cubano se expande alrededor del mundo en lucha humanitaria contra la pandemia (AUZA; DORTA, 2020; GUANCHE, 2020a; GUANCHE, 2020b; RAMOS; AMBROSIO, 2020).

A nivel mundial, diversos países a consecuencia de la pandemia han reconocido que el sistema de salud precisa mayor inversión para su fortalecimiento, porque pareciera que los esfuerzos realizados para detener la crisis fueran en vano. Sin embargo, debe recordarse que 
la implementación de políticas públicas dependerá de la capacidad económica de cada país para llevarlas a cabo, pues no siempre se pueden implementar acciones sin obstáculos, pese a observarse consecuencias en el sistema de salud y sus profesionales, como ocurre en el contexto actual. En el caso de México, señalan Auza y Dorta, 2020 que la crisis mundial hace evidente: la escasa inversión que se tenía en salud, su dependencia del extranjero para suministro de insumos y equipo médico, situación que en dicho país ha propiciado la iniciativa para la fabricación de equipos de protección, caretas y otros recursos. En España, estudios muestran que a partir del caos generado a raíz de la pandemia se ha creado conciencia sobre la desatención existente en el sistema y con el personal de salud, de igual manera se hace evidente la escasez de recursos para atender casos especiales como los presentados en el contexto de salud actual, se indica también que el fortalecimiento de la salud pública implica además de enseñanzas de tipo teóricas el acercamiento a la realidad social por parte de los futuros profesionales de la salud (HERNÁNDEZ y GARCÍA, 2021)

Por su parte, en Cuba, el Ministerio de Salud Pública con el fin de afrontar la crisis y ayudar a la población, actualiza información de la situación internacional y nacional de la epidemia, además de promover la prevención en la población, tanto que los principales retos de los profesionales de salud en cuba han sido el cuidado de la población, control y prevención del contagio en la comunidad y a nivel institucional (GUANCHE, 2020a). Desde Cuba, se ha propuesto que cada país invierta en la salud de manera sólida y administrar los recursos de forma adecuada (AUZA; DORTA, 2020). Actualmente, hasta el 16 de abril del año en curso se reporta que Cuba estaba preparada previamente a la crisis, esto le mantiene firme en su sistema de salud, sin colapsos, aunque con riesgos, de tal forma que, cuenta con reservas de camas, laboratorios y servicios de salud disponibles.

Para el caso de Inglaterra, ante el reconocimiento de la debilidad del sistema de salud se proponen estrategias para su fortalecimiento, especialmente encaminadas hacia el bienestar humanitario, tales como: la revisión estructural y funcional del sistema, ampliación de la capacidad laboral e incremento de sueldos, garantía de apoyo a quienes requieran vivir independientemente, ampliar el personal de atención también en entornos comunitarios, garantizar a los pacientes dados de alta lugares alternativos al hogar como mecanismo de prevención del contagio, dotación de insumos hospitalarios y cuidar de manera especial a los trabajadores de la salud (RUANE, 2020). La anterior reforma es con el fin de responder a los impactos psicológicos asociados con agotamiento físico y mental experimentado por diversos profesionales (médicos, enfermeros, odontólogos, etc.) en el desarrollo de sus funciones (URZUA et al., 2020; VALERO, 2020). Factores que no solo afectan al personal del área de salud de Inglaterra, sino que resultan ser detonantes de los problemas de salud padecida por el profesional sanitario a lo largo de los continentes, como lo presenta este documento.

\section{Condiciones de trabajo y efectos en la salud de los profesionales en el afrontamiento del Covid-19}

A propósito del reconocimiento que hace Inglaterra sobre las prácticas profesionales en el marco de la crisis actual, vale la pena citar los planteamientos de autores como Fuentes, 2020, señalando que el secretario general de la Organización de las Naciones Unidas, el Día Mundial de la Salud 2020, indicó que en la mayoría de países el profesional de salud labora de manera intensa y en escasez de equipos de protección personal. Lo anterior ha sido una de las 3 causantes de la propagación del virus en el personal que labora en el área de salud (Foro Internacional FIMI, 2020): 
1. La primera causa reportada es la falta de entrenamiento para enfrentar crisis provocadas por virus nuevos.

2. La sala de espera del área de salud puede ser frecuentada por usuarios asintomáticos, que consultan por otro tipo de dolencias. Adicionalmente, es común encontrar en las instituciones de salud Latinoamericanas salas congestionadas con usuarios que esperan largas horas para ser atendidos, lo que en consecuencia resulta ser factor de riesgo para la propagación del virus.

3. En tercer lugar, la FIMI, 2020 reporta que muchos países ante sus bajos recursos económicos no pueden acceder a los múltiples trajes de bioseguridad (de tipo desechable) que necesita cada profesional sanitario, adicionalmente, la calidad de los trajes ha sido reducida, el precio incrementado y el personal que labora en el área de salud en variadas ocasiones ha accedido al equipo de seguridad con sus propios recursos monetarios.

Por ahora, un ejemplo de lo anterior lo reporta una investigación realizada en Ecuador con 85 enfermeros que laboran en un hospital, cuyos resultados arrojaron que un factor de gran impacto en la salud, es no tener la cantidad suficiente de suministros de protección como mascarillas, gafas, guantes, gel alcohol, entre otros, lo que dificulta cumplir los protocolos de bioseguridad (BARRERA, 2020). Como se podrá apreciar, la situación anterior se presenta a largo de los continentes.

En este orden de ideas, respecto a Latinoamérica, se ha experimentado en el transcurso de la pandemia un fuerte impacto epidemiológico, debido a la precariedad en el sistema de salud, desigualdad social, falta de capacitación a personal de salud, personal altamente vulnerable por su cercanía a pacientes contagiados y tener a su vez contacto con otros entornos sociales y dentro del mismo hospital (BRIONES et al., 2020). Esta situación también les hace vulnerables a la estigmatización social en algunos contextos, a tal punto que han sido víctimas de agresiones por desencadenar representaciones sociales asociadas a "peligro, personal contagiador de Corona Virus".

Lo anteriormente mencionado, se refleja en el reporte de diversos autores, en el que en varios países indican que el profesional de salud ha sido víctima de episodios violentos: en México, se reporta el mayor número de casos de agresión ciudadana al personal del área de la salud, una enfermera fue agredida con un líquido caliente y un médico con cloro; en una ciudad, no reportada, de Colombia, a una enfermera le arrebataron el tapabocas y lesionaron su piel con vidrio (ARENAS; MONTOYA; VELÁSQUEZ, 2020); en Medellín, un médico fue agredido por familiares de un paciente que contrajo Covid (ARENAS; MONTOYA; VELÁSQUEZ, 2020); en Cartagena otro profesional recibió impacto en su cabeza con objeto pesado, porque informó a la familia de un paciente que este debía ser aislado (ARENAS; MONTOYA; VELÁSQUEZ, 2020); en Nueva York médicos y enfermeras han dormido en sus coches, porque la sociedad los percibe como fuentes de transmisión infecciosa (FIMI, 2020).

Los casos de discriminación se han presentado de igual manera en otros países Latinoamericanos (ARENAS; MONTOYA; VELÁSQUEZ, 2020), tales como Argentina, Brasil, Bolivia, España y La India (BEDOYA, 2020). En Costa Rica 1 caso reportado, en Perú se encontraron 2 situaciones, panamá reporta 4 casos de discriminación, Venezuela 9, en Argentina 10, Colombia 20 casos y en México se reportan un total de 40 casos (FIMI, 2020). Todos son casos aislados, causados por el temor de la población a ser contagiada, lo que a su vez genera temor en los equipos de salud (BEDOYA, 2020), no obstante, la FIMI, 2020 reporta que casos de estigmatización sufridos por el personal de la salud existían previamente a la pandemia, tan solo que estaban invisibilizados. 


\section{Efecto en la salud mental del personal de salud como consecuencia de los retos afrontados.}

Lo anterior cobra relevancia a partir de investigaciones realizadas a lo largo de diversos continentes, en países como Paraguay, Chile, Brasil, Colombia, China, Rumania, Alemania, Ecuador e Italia. Así para empezar tomemos el caso de Paraguay, los profesionales sanitarios protestaron reclamando abastecimiento de equipos de seguridad, horarios de trabajo justo, factores que les predisponía para padecer la denominada fatiga por compasión (SAMANIEGO et al., 2020), pues sus jornadas laborales se habían extendido por exceso de número de pacientes por profesional, afrontaban además muertes constantes de usuarios (SAMANIEGO et al., 2020).

Otros estudios realizados en este país confirmaron la presencia síntomas de depresión (44\%) y síntomas de ansiedad (43\%) en 90 trabajadores del área de medicina y enfermería (BETANCOURT et al. 2006, como se citó en Samaniego et al., 2020). De manera similar el estudio de Samaniego et al. (2020) evaluó síntomas relacionados con afectación de la salud mental en 126 profesionales sanitarios de medicina, enfermería, psicología y odontología entre otras. El 32,2\% del personal encuestado presentaba afectación por depresión, el 41,3\% ansiedad, el 27,8\% problemas de insomnio, el 38,9\% distrés y 64,3\% fatiga por compasión, uno de cada 3 profesionales padecía los síntomas y estos se asociaban a las exigencias a que se sometían en su rol profesional.

En Chile, de un total de 125 trabajadores de la salud, encuestados, el 62,4\% carecían de equipos de protección individual, 65,6\%, experimentaban síntomas depresivos, 74,4\% padecían de ansiedad, el 64,8\% tenían problemas de insomnio y el 56,8\% distrés, siendo los profesionales que tienen contacto directo con infectados los que presentan, en mayor medida, sintomatología negativa en su salud mental (URZÚA et al., 2020).

Prosiguiendo con Brasil, al igual que en la mayoría de países, los profesionales de la salud tan solo a inicios de la pandemia ya presentaban cansancio físico y mental, dificultades en la toma de decisiones frente a pacientes que sufrían complicaciones; ansiedad ante diversos fallecimientos de pacientes, colegas y familias, adicionalmente, intranquilidad por cierre de escuelas y escases de equipos de protección individual (EPI) los cuales deben ser política de estado (MEDEIROS, 2020). En el nombrado país ha sido también un reto contratar personal cualificado especialmente para la unidad de terapias intensivas, sugiriéndose abastecer el área tanto a nivel de insumos como con capital humano (MEDEIROS, 2020). Pese a haber transcurrido más de un año de inicio de la crisis permanecen los retos y el impacto en el estado de ánimo del profesional de salud Brasileño, así lo corroboran Ramos et al. (2021) en un estudio realizado en Paraná, cuyo objetivo era comprender los sentimientos de trabajadores que atienden pacientes con sospecha de Covid-19. Los investigadores encontraron que prevalecían sentimientos de ambivalencia, temor, ansiedad, preocupación ante la muerte, discriminación e incertidumbre acerca del futuro. Esta población es altamente vulnerable para desestabilizarse emocionalmente, porque deben enfrentar sus sentimientos de impotencia, fracaso, estrés por condiciones laborales precarias, incertidumbre sobre la cura, infectarse o infectar, pérdida de pacientes, desgaste mental y físico (BORGES et al., 2020; SOARES; PEDUZZI; VIANA, 2021).

En el caso específico de Colombia, el personal de salud realiza sus funciones laborales bajo presión (MÁRQUEZ 2020), este y otros factores son causa de un fuerte impacto en la salud mental, así lo corrobora la literatura revisada por Arenas; Montoya y Velásquez (2020), quienes hallaron un estudio sobre salud mental en personal de salud (médicos 67\%, enfermeros 22\% y otros profesionales 9\%), a partir de 711 encuestas. El estudio arrojó como resultado que un 33.3\% presentaban ansiedad, $26.3 \%$ depresión, $11.7 \%$ insomnio, siendo los médicos y enfermeras 
el personal afectado en mayor medida. La depresión, la ansiedad y el insomnio tienen sus raíces en factores como el distanciamiento de la familia para no contagiarles, la sobre carga laboral, la discriminación social, todo ello ocasiona estrés psicológico o síndrome de desgaste profesional (ARENAS; MONTOYA; VELÁSQUEZ, 2020).

Hallazgos similares se encuentran en el estudio realizado por Monterrosa et al., 2020, en el que participaron 541 médicos Colombianos, los investigadores informan sobre elevados índices de ansiedad, estrés y miedo padecido por el personal. El 39\% de los médicos se han sentido discriminados por laborar en el área de salud; más del 70\% indicaron sentir miedo por contagio, morir, llegar a ser transmisores o ser asintomáticos; 6\% padecía estrés laboral, 72,9\% manifestó síntomas de ansiedad y solicitaron abastecimiento de elementos de trabajo. El estudio realizado por Mera et al. (2020) en hospitales de nivel III de atención, en la ciudad Popayán (Colombia), tuvo como objetivo conocer las necesidades de elementos de protección personal (EPP) en 521 personas que laboran en el área de salud. Los investigadores hallan que el $47.9 \%$ no habían sido capacitados para el uso de EPP, luego de ser declarado el estado de emergencia; el 37\% informan no tener conocimiento sobre el uso de EPP; 64.5\% compraron con recursos propios sus EPP (especialmente, tapabocas, mascarilla quirúrgica, mascarilla N95), haciéndose evidente la necesidad tanto de capacitación como de elementos de protección.

Por lo anterior, el personal de salud colombiano ha expresado que además de felicitaciones requieren equipos de protección e insumos para hacer un trabajo eficiente (ARENAS; MONTOYA; VELÁSQUEZ, 2020). Se observa así que no se está trabajando con apropiadas condiciones de bioseguridad, la infraestructura es deficiente e insuficiente el capital humano en las Instituciones Prestadoras de Servicios (VELÁSQUEZ, 2020). Abastecer del equipo de protección adecuado al personal que labora en el área de salud debe ser una ley universal, especialmente porque mientras se establece cuarentenas y órdenes que obligan a la población a permanecer en sus casas, los profesionales de la salud deben proseguir con la atención de pacientes (FIMI, 2020).

Haciendo revisión sobre los retos que afronta el profesional de la salud y el impacto en su bienestar, para el caso de China se encuentra que la mayor presión laboral que experimentan es debido al uso inadecuado de equipo de protección, alto riesgo de infectarse, excesiva sobrecarga de actividades y pérdida de contactos personales, con serias consecuencias sobre su salud mental (LOZANO, 2020a). En este sentido, según cifras de un estudio realizado en China el 39\% de los encuestados padecían impactos psicológicos con síntomas de depresión o ansiedad, siendo los médicos y enfermeras que atienden en primera línea a pacientes infectados los más perjudicados (URZUA et al., 2020), adicionalmente, eran 3.300 los casos de personal de la salud infectados (MÁRQUEZ, 2020).

Otro estudio realizado con 1257 trabajadores de la salud de 34 hospitales de China, arrojó que el personal manifestaba síntomas de Distrés 71.5\%, ansiedad 44.6\%, depresión 50,4, síntomas de insomnio 34\% con mayor impacto en mujeres enfermeras (BUENO y BARRIENTOS, 2020; JUÁREZ, 2020; MARTÍNEZ, 2020). Otros estudios, también en China, sobre el tema han proporcionados bases teóricas para la intervención del impacto y sufrimiento psicológico, al encontrar entre los encuestados síntomas de estrés, ansiedad, depresión y detectar factores de riesgo y protección ante el estrés (LOZANO, 2020a). El personal de cuidados intensivos llega al límite del estrés cuando personas adultas mayores no pueden despedirse de sus seres queridos (VALERO, 2020).

Retos afrontados por el personal de salud en el marco de la pandemia y sus consecuencias en el bienestar psicológico también han sido reportado en Rumania, Alemania, China, donde investigadores identificaron que el personal de salud ha padecido en mayor medida síndrome 
de Burnout (agotamiento emocional, despersonalización, sentirse con baja realización personal), quizá a consecuencia de las amenazas que representa el Covid-19 y los turnos nocturnos en incremento, (LOZANO, 2020b). El Burnout es un efecto psicológico generado por estresores crónicos del contexto laboral, no debe aplicarse a experiencias provenientes de otras áreas (JUÁREZ, 2020). Tal síndrome, entre otros, también se encontró en Ecuador y en México, como lo evidencian los siguientes datos de estudios realizados.

Peraza, 2020, reporta que Ecuador, se ha encontrado entre los 3 países de Latinoamérica con más casos de Covid confirmados y la cantidad de personal de salud afectada (médicos, enfermeros, odontólogos, psicólogos etc.), especialmente por falta de equipo de protección personal limita la atención de pacientes. En una clínica privada ubicada en la ciudad de Cuenca 78 personas fueron encuestadas, el 25.6\% indican síntomas de agotamiento emocional, el 17.9\% reportaron sentimientos de despersonalización y el $24.4 \%$ baja realización personal, todo asociado a diversos factores, entre ellos la presión laboral, constantes fallecimientos presenciados, riesgo al contagio, todo ello desestabiliza emocional y físicamente al personal de salud (PARRA, 2020). El Burnout en médicos de Ecuador se ha asociado a la carga laboral y ejecución de funciones en contextos contaminados (URGILÉS, 2020).

En México un estudio con personal de salud que ejerce funciones en diversos hospitales, halló que un $47 \%$ dice necesitar apoyo psicológico, 27,4\% presentó síntomas de ansiedad, 16,9\% padecía depresión, 44,1\% manifestó estrés, 47,6\% desgaste emocional o Burnout (JUÁREZ, 2020). Burnout es una epidemia invisibilizada en países en vías de desarrollo, por la alta demanda asistencial que representa (MACAYA; ARANDA, 2020). Similar a lo ocurrido en Ecuador, en Italia, el virus se propagó a 17.000 trabajadores de la salud, entre ellos enfermeras, médicos, obstetras, trabajadores sociales, pediatras, especialmente porque trabajaban en diversas instituciones sin el equipo de protección personal adecuado y débiles medidas de protección entre los trabajadores de la salud (VALERO, 2020).

Resultaría pertinente la contratación de más profesionales de la salud, como estrategia para controlar el elevado impacto adverso que en la salud se ha generado por la sobrecarga laboral. La Organización Mundial de la Salud (OMS, 2020) para el caso de la enfermería, ha indicado que en todo el planeta, el personal de salud lo constituye 43,5 millones de profesionales, siendo el $50 \%$ personal de enfermería y a excepción de Brasil, Islandia y Nueva Zelanda, se estima menos de 3 profesionales de enfermería por cada 1000 habitantes, lo que los deja expuestos a alta carga laboral e incremento de usuarios contagiados para su atención, aumentando así su nivel de estrés (FUENTES, 2020).

En el caso del profesional en Fisioterapia, un estudio ha mostrado su importante rol en la intervención con pacientes con Covid-19. Las diversas fases en las que el profesional interviene incluyen también ejercicios físicos y de respiración: Fase de aislamiento, hospitalización, terapia intensiva, recuperación y alta (PEREIRA et al., 2020). Cuando se trata de pacientes que ingresan a cuidados intensivos, por Covid-19, el fisioterapeuta tiene un importante rol en la restauración de la función pulmonar y en tratar la debilidad muscular del paciente crítico, por tanto, se requiere personal con experticia en el ámbito cardiorrespiratorio y que se le garantice el protocolo de seguridad (LISTA et al., 2020). En síntesis, el personal de salud por la atención directa con los enfermos, debe tener capacitación permanente (PEREIRA et al., 2020), siendo su bienestar un requisito fundamental, para la adecuada asistencia a la población (ARENAS et al., 2020).

En lo correspondiente al profesional de psicología, se ha señalado que los profesionales psicólogos que hacen parte del equipo de salud frente al Covid-19 tienen grandes desafíos, como 
lo es la exposición directa al paciente infectado, insuficiente material de protección, recurrente apoyo psicológico a situaciones de luto, prevención y promoción de la salud mental, es por tanto un contexto que también exige cuidado a estos profesionales (GRINCENKOV, 2020).

Aunque comúnmente se ha reconocido que la trasmisión del virus en el personal de salud, pueda deberse a no portar el equipo de protección adecuado cuando se acerca a pacientes infectados, la infección no siempre es por contacto con pacientes, así se evidenció en un Hospital de Taipéi, en el que 17 profesionales de la salud padecieron SARS, aún sin contacto directo con pacientes, el RNA del virus se encontraba en superficies limpias (ARENAS et al., 2020).

Antes de finalizar y en función de lo reportado previamente, resulta pertinente recopilar algunas de las estrategias que diversos autores desde sus respectivos países han propuesto o implementado, para mejorar tanto el sistema de salud como el bienestar del profesional, al reconocer las falencias presentadas en el adecuado manejo de una imprevista pandemia. Arenas et al. 2020, ha señalado lo fundamental de capacitar sobre el manejo de equipos de protección personal y resalta que un mecanismo de protección profesional, es controlar el acceso de profesionales de salud a las instalaciones y el número de profesionales por paciente. Gonzales et al. 2020, recomienda aplanar la curva de contagio mediante prácticas de higiene sanitaria y el aislamiento de infectados, además de invertir en programas con énfasis en la investigación de la salud. Briones et al. 2020, también le apuesta a las prácticas de higiene, mediante el uso de luz UV-C como método de desinfección de superficies, al destruir el ADN y ARN de bacterias, virus y patógenos, disminuyendo así el riesgo de contagio entre los profesionales. Autores como Márquez (2020) recomiendan el uso de la telemedicina como método de reducir la carga laboral del personal de salud.

Kershenobich (2020), inspirado en el contexto Mexicano, refiere que la pandemia ha revelado la necesidad de preparar al profesional y fortalecer la investigación para superar futuros y actuales retos. Similarmente Ramos y Ambrosio (2020), plantean cambios en la enseñanza de la medicina que incluya la pesquisa, labores asistenciales, capacitación en la prevención de infecciones, comunicación sobre resultado de investigaciones científicas. Autores como Fuentes (2020) enfocan sus propuestas en el bienestar del personal de enfermería, señalando que podrían tener más remuneración salarial, estímulos y capacitación mediante formación avanzada. Especialistas Latinoamericanos del área clínica, han solicitado a organizaciones gubernamentales de derechos humanos y a las autoridades con compromiso multinacional, la organización internacional del trabajo (FIMI, 2020): 1) Dotar los centros de atención para los pacientes con los debidos insumos; 2) Que en caso de fallecimiento del médico su familia sea acreedora de beneficios como educación, salud y subsistencia económica; 3) Garantizar reparación de daños físicos o materiales en caso de sufrir agresión; 4) Que al personal de entrenamiento no les extiendan la jornada laboral o que se les remunere debidamente.

\section{Consideraciones Finales.}

El presente trabajo se ha centrado en los retos que afronta el personal que labora en el área de salud en diversos contextos mundiales, en el marco de la pandemia por Covid-19, su impacto a nivel psicológico y las estrategias optadas por salud pública de cada país ante la situación, resaltando que el virus no solo ataca a nivel físico que es el mayor énfasis que surge alrededor de la pandemia, sino que también debe abordarse la situación desde una perspectiva biopsicosocial o integral. A nivel mundial la problemática social se manifiesta principalmente 
a través de síntomas asociados con depresión, estrés, insomnio, ansiedad y solo 3 autores de toda la literatura revisada reportaron casos de temor ante posible agresión por estigmatización social hacia ese personal. Tal situación de salud mental en los profesionales posiblemente existía previa a la pandemia, no obstante, se incrementó y detonó desde el inicio de la crisis, afectando en mayor medida a mujeres en comparación con el género masculino.

El síndrome de Burnout, pese a ser considerado por la OMS como consecuencia de la exposición a estresores crónicos provenientes del contexto laboral y que impactan fuertemente la salud mental, en la bibliografía revisada, únicamente se encontró en países como Rumania, Alemania, China, México y Ecuador. Lo anterior llama la atención porque, sin duda, en el contexto de pandemia actual son numerosos y perjudiciales los retos que los profesionales de la salud deben afrontar y entre ellos uno muy revisado y manifestado por los trabajadores sanitarios es el estrés laboral con los factores que lo componen, por ejemplo, el reto de atender en plenitud un contexto de salud saturado, reto del que se ha hecho poco énfasis, y no tener equipos apropiados para realizar la atención. La falta de hallazgos sobre dicho síndrome quizá se deba a la falta de investigaciones sobre el tema, no obstante, sea bajo categorías diagnósticas o no, los síntomas asociados al Burnout pueden existir en el personal de salud, a tal punto de salirse de control si no es tratado a tiempo ¿Cuáles serían las consecuencias sociales si todo el personal de salud se incapacitara? ¿La falta de abastecimiento de EPP es por falta de recursos económicos o porque se está normalizando la situación?

Para finalizar, se espera que el personal que labora en contacto directo contra el Covid-19 sea atendido en sus necesidades vitales, los determinantes sociales deben afectar favorablemente el bienestar físico mental y social de la población y la comunidad profesional, además de ser prioritaria su identificación para aminorarlos. Esto resultaría de enorme bienestar para la salud mental que, de acuerdo a las cifras reportadas, ha sido impactada significativamente. Se debe implementar además de acciones desde salud mental que resultan fundamentales, también estrategias para la atención de las necesidades inmediatas y de protección Biopsicosocial.

\section{Referencias.}

ARENAS, M. D. et al. Protección de los profesionales sanitarios en Nefrología ante la pandemia por COVID-19. Nefrología, 395-402, 4 de jul-August 2020. DOI: https://doi.org/10.1016/j.nefro.2020.06.001.

ARENAS, L.M.; MONTOYA, M.; VELÁSQUEZ, K. Coronavirus (covid-19) y su Impacto en la Salud Mental de los Profesionales de la Salud en Antioquia, septiembre, 2020. Monografía (Especialista en Gerencia de la Calidad y Auditoría en Salud). Facultad de Ciencias Económicas y Administrativas, Universidad Cooperativa de Colombia, Medellín, 2020.

AUZA, J.; DORTA, J.A. La COVID-19 y los desafíos que impone para el profesional de la salud en Cuba. Revista Cubana de Investigaciones Biomédicas, Cuba, 1-5, 3 de julio de 2020. Disponible en: http:// www.revibiomedica.sld.cu/index.php/ibi/article/view/836/888.

BARRERA, T. A. Aplicación de normas de Bioseguridad en el personal de enfermería en tiempos de pandemia en el hospital Basico Pelileo en el periodo marzo - noviembre 2020. Monografía (Graduación en enfermería). Facultad de ciencias de la Salud, Universidad Técnica de Ambato, Ecuador marzo, 2021 Disponible en: http://repositorio.uta.edu.ec/handle/123456789/32640 Acceso en: 11 mayo 2021

BEDOYA, C.M. Covid-19: La pandemia del maltrato contra el personal de la salud en tiempos de pandemia. JEPH, p. 1-3, 6 de junio/2020. DOI: https://doi.org/10.18041/2665-427X/. 
BORGES, M. et al. Intervenções em saúde mental para profissionais de saúde frente a pandemia de Coronavirus. Rev enferm UERJ, Rio de Janeiro, p. 1-6, mayo/2020. DOI: http://dx.doi.org/10.12957/ reuerj.2020.49923.

BRIONES, N. et al. Luz ultravioleta para desinfección en áreas de salud, frente al covid-19. Revisión de literatura. Revista OACTIVA, 1-8, Diciembre/2020. DOI: https://doi.org/10.31984/oactiva.v5i3.501.

BUENO, M.; BARRIENTOS, S. Cuidar al que cuida: el impacto emocional de la epidemia de coronavirus en las enfermeras y otros profesionales de la salud. Enferm Clin. 1-5, Mayo de 2020. DOI: https://doi. org/10.1016/j.enfcli.2020.05.006.

Foro Internacional de Medicina Interna (FIMI), 2020. Ataque al personal de la salud durante la pandemia de COVID-19 en Latinoamérica. Acta Médica Colombiana Vol. 45 N³ Julio-Septiembre 2020 DOI: https://doi.org/10.36104/amc.2020.1975.

FUENTES, G. P. Enfermería y COVID-19: reconocimiento de la profesión en tiempos de adversidad. Rev. Colomb. Enferm. p. 1-4, 4-4-2020. DOI: https://doi.org/10.18270/rce.v19i1.2970.

GONZALES, J. et al. Pandemia de la COVID-19 y las Políticas de Salud Pública en el Perú. Revista de Salud Pública, 1-9, 30 abril/2020. DOI: https://doi.org/10.15446/rsap.V22n2.87373.

GRINCENKOV, F.R. A Psicologia Hospitalar e da Saúde no enfrentamento do coronavírus: necessidade e proposta de atuação. HU Rev. Juiz de Fora, 13-21. 8 de abril de 2020. DOI: https://doi. org/10.34019/1982-8047.2019.v45.16970.

GUANCHE, H. COVID-19. Un reto para los profesionales de la salud. Rev haban cienc méd, La Habana Cuba, p. 1-4, abril, 2020a. Disponible en: http://www.revhabanera.sld.cu/index.php/rhab/article/ view/3284/2484.

GUANCHE, H. COVID-19. La necesidad de nuevos paradigmas de cooperación y riesgo de los trabajadores de la salud. Rev haban cienc méd, 1-5, 2020b. Disponible en: http://www.revhabanera. sld.cu/index.php/rhab/article/view/3456.

HERNÁNDEZ, A.; García, A.M. ¿Será mejor la salud pública tras la COVID-19? Gac Sanit. España, 1-2, https://doi.org/10.1016/j.gaceta.2020.06.004.

JUÁREZ, A. Síndrome de Burnout en personal de salud durante la pandemia COVID-19: un semáforo naranja en la salud mental. Salud UIS, México, 432-439, diciembre de 2020. DOI: https://doi. org/10.18273/revsal.v52n4-2020010.

KERSHENOBICH, D. Fortalezas, deficiencias y respuestas del sistema nacional de salud frente a la Pandemia del Covid-19. Rev. Economíaunam, 1-6, Dic, 2020. DOI: https://doi.org/10.22201/ fe.24488143e.2020.51.545.

LANDEROS, E.; ARROYO, F.J.; RODRÍGUEZ, M. de J. Formulación de políticas públicas en salud durante la pandemia de covid-19 en países americanos. SANUS, 1-10, Octubre- diciembre/2020. DOI: https://doi. org/10.36789/sanus.vi16.227.

LAZCANO, E. Alfabetización en salud pública ante la emergencia de la pandemia por Covid-19. Salud pública de México, 1-10, 8 de mayo de 2020. DOI: https://doi.org/10.21149/11408.

LISTA, A.; GONZÁLEZ, L.; SOUTO, S. ¿Qué papel desempeña la Fisioterapia en la pandemia Mundial por COVID-19? Fisioterapia 2020, 1-5. DOI: https://doi.org/10.1016/j.ft.2020.04.002.

LOZANO, A. Impacto de la epidemia del Coronavirus (COVID-19) en la salud mental del personal de salud y en la población general de China. Rev Neuro psiquiatría, Lima, p. 51-56, enero 2020a. 
Disponible en http://www.scielo.org.pe/scielo.php?script=sci_arttext\&pid=S0034-85972020000100051. accedido en 9 mayo 2021.

Lozano, A. El síndrome de Burnout en los profesionales de salud en la pandemia por la COVID-19. Rev Neuropsiquiatr. 1-2, 2020b. DOI: https://doi.org/10.20453/rnp.v84i1.3930.

MACAYA, P.; ARANDA, F. 2020. Cuidado y autocuidado en el personal de salud: enfrentando la pandemia COVID-19. Rev Chil Anest, 356-362, 2020. DOI: https://doi.org/10.25237/ revchilanestv49n03.014.

MÁRQUEZ, J. Teleconsulta en la pandemia por Coronavirus: desafíos para la telemedicina pos-COVID-19 Rev Colomb Gastroenterol, 1-12, Dec-2020. DOI: https://doi.org/10.22516/25007440.543.

MARTÍNEZ, S. (2020). Necesidad de apoyo psicológico en profesionales sanitarios ante el COVID-19. Psiquiatría Biológica, 1-2, diciembre de 2020. DOI: https://doi.org/10.1016/j.psiq.2020.08.001.

MEDEIROS EA. A luta dos professionais de saúde no enfrentamento da COVID-19. Acta Paul Enferm, 1-4, maio 2020, Disponible en: https://www.scielo.br/j/ape/a/Nc8yzcvtrvXbWBgBGskm36S/?lang=pt

MERA A. et al. Conocimientos y necesidades del personal de salud sobre elementos de protección personal en el departamento del Cauca, durante la pandemia por coronavirus COVID-19. OSF Preprints. Resultados preliminares. 2020. p. 1-23. Disponible en: https://osf.io/7agph.

MONTERROSA, A. et al. Estrés laboral, ansiedad y miedo al COVID-19 en médicos generales colombianos. MedUNAB, 195-213, junio, 2020. DOI: https://doi.org/10.29375/01237047.3890.

PARRA, J.C. Síndrome de Burnout y relación con los factores sociodemográficos y laborales en profesionales de la salud en una clínica privada de la ciudad de Cuenca en el contexto con la emergencia sanitaria por COVID-19. 2020. Disertación (Magister en Salud Ocupacional y Seguridad en el Trabajo). Universidad de Azuay, Cuenca-Ecuador, 2020. Disponible en: http://dspace.uazuay.edu. ec/handle/datos/10411 Acceso en: 8 mayo. 2021

PERAZA, C. X. Salud laboral frente a la pandemia del COVID-19 en Ecuador. Medisur, p. 507-511, may. 2020. Disponible en: <http://medisur.sld.cu/index.php/medisur/article/view/4713, Fecha de acceso: 06 may. 2021

PEREIRA, J. et al. Fisioterapia y su reto frente al COVID-19. Scielo Preprints, 1-14, 24-04-2020. DOI: https://doi.org/10.1590/SciELOPreprints.157.

RAMOS, A.; AMBROSIO, R. Actualidades de la educación médica cubana frente a la COVID-19. Rev Cubana Invest Bioméd, Ciudad de la Habana, p. 1-4, sept. 2020. Disponible en http://scielo.sld.cu/ scielo.php?pid=S0864-03002020000300026\&script=sci_arttext\&tlng=en.

RAMOS, A.C. et al. Reações e sentimentos dos profissionais de saúde no cuidado de pacientes hospitalizados com suspeita de covid-19. Rev Gaúcha Enferm., p. 1-7, 2021. DOI: https://doi. org/10.1590/1983-1447.2021.20200160.

RUANE, S. Próximos pasos para fortalecer los Sistemas de Salud y Atención Social del Reino Unido a partir de las debilidades reveladas por la pandemia de coronavirus. Salud internacional, 1-3, marzo 2020. Disponible en: https://dialnet.unirioja.es/servlet/articulo?codigo=7524929.

SAMANIEGO, A., URZÚA, A., BUENAHORA, M., \& VERA-VILLARROEL, P. Sintomatología asociada a Trastornos de Salud Mental en trabajadores sanitarios en Paraguay: Efecto COVID-19. Revista Interamericana de Psicología, p. 1-19. 2020. https://scholar.google.com/scholar?cluster=1737724808 8587443745\&hl=es\&as_sdt=0,5. 
SOARES, C.; PEDUZZI, M. VIANA, M. Os trabalhadores de enfermagem na pandemia Covid-19 e as desigualdades sociais. Rev Esc Enferm USP, 1-3, 2020. DOI:https://doi.org/10.1590/S1980220X2020ed0203599.

URGILÉS, S. TÍTULO: Sindrome de Burnout en el personal de salud que trabaja en la unidad de terapia intensiva durante la pandemia COVID-19 en un hospital de Quito. Disertación (Maestría en Ergonomía Laboral). Facultad de Ciencias, Universidad Internacional SEK, Quito, 2020. Disponible en: https://repositorio.uisek.edu.ec/handle/123456789/3970. Acceso en: 2 mayo. 2021

URZUA A. et al. Salud mental en trabajadores de la salud durante la pandemia por COVID-19 en Chile. Rev. Méd. Chile, Santiago, 1121-1127, Agosto 2020b. DOI: http://dx.doi.org/10.4067/S003498872020000801121.

VALERO, N. La bioseguridad y el personal de salud: a propósito de la pandemia de covid-19. Enfermería Investiga, p. 1-4, jun. 2020. ISSN 2550-6692. Disponible en: https://revistas.uta.edu.ec/erevista/index. php/enfi/article/view/901. Acceso: 09 mayo 2021.

Velásquez, C.J. Enfermería en Colombia en tiempos de la pandemia por coronavirus. Revista Ciencia y Cuidado, Cúcuta, 5-7, Agosto, 2020. Disponible en: https://dialnet.unirioja.es/servlet/ articulo?codigo $=7490965$. 